\title{
Single-photon photoionization of oxygen-like Ne III
}

\author{
S. N. Nahar ${ }^{\mathrm{a}}$, A. M. Covington ${ }^{\mathrm{b}}$, D. Kilcoyne ${ }^{\mathrm{c}}$, V. T. Davis ${ }^{\mathrm{b}}$, J. F. \\ Thompson $^{b}$, E. M. Hernández ${ }^{\mathrm{d}}$, A. Antillón ${ }^{\mathrm{d}}$, A. M. Juárez ${ }^{\mathrm{d}}$, A. \\ Morales-Mori $^{\mathrm{d}}$, G. Hinojosa ${ }^{1 \mathrm{~d}}$ \\ ${ }^{a}$ Department of Astronomy, The Ohio State University, OH 43210-1173, USA \\ ${ }^{b}$ Physics Department, University of Nevada, Reno NV 89557-0220, USA \\ ${ }^{c}$ The Advanced Light Source, Lawrence Berkeley National Laboratory, CA 94720, USA \\ ${ }^{d}$ Instituto de Ciencias Físicas, Universidad Nacional Autónoma de México, A. P. 48-3, \\ Cuernavaca 62251, Mexico
}

\begin{abstract}
We offer a theoretical and experimental study of the single-photon photoionization of Ne III. The high photon flux and the high-resolution capabilities of the Advanced Light Source at the LBNL were employed to measure absolute photoionization cross sections. The resulting spectrum has been benchmarked against high accuracy relativistic Breit-Pauli $R$-matrix calculations. A large close-coupling wave function expansion which comprises up to 58 fine-structure levels of the residual ion Ne IV of configurations $2 s^{2} 2 p^{3}, 2 s 2 p^{4}$, $2 p^{5}, 2 s^{2} 2 p^{2} 3 s, 2 s^{2} 2 p^{2} 3 p$ and $2 s^{2} 2 p^{2} 3 d$ was included. A complete identification of the measured features was achieved by considering seven low-lying levels of Ne III. We found that the photoionization cross-section $\left(\sigma_{P I}\right)$ exhibits the presence of prominent resonances in the low-energy region near the ionization thresholds that correspond to low-lying levels. These include high-peak narrow resonances with almost zero background introduced by relativistic effects. However, there does not exist a significant contribution to $\sigma_{P I}$ from relativistic effects at the high-energy interval of the present study.
\end{abstract}

Keywords:

Photoionization, Isoelectronic to oxygen, Opacity, Auto-ionizing states, Synchrotron radiation, Soft X-rays, Breit-Pauli, R-matrix, ab-initio PACS: 31.15.A- 31.30.jc, 32.30.Rj, 32.70.Fw, 32.80.Ee, 32.80.Fb, 32.80.Zb, 95.30.Ky, 97.10.Ex 


\section{Introduction}

There is a growing interest in advancing the present knowledge about the photoionization (PI) of ions due to the wide range of applications in which the interaction of ions with radiation is of relevance. For example, in plasma science, autoionizing states induced during PI are proposed as a source of thermal electrons [1]. Knowledge of the autoionization energies is critical in the derivation of charge-state distributions in plasmas [2]. This knowledge is of importance in the opacity problem which is paramount in astrophysics [3]. The search for life-propitious regions in the Universe [4], the study of starformation [5] and the understanding of certain atmospheric environments [6] rely also, at least in part, on the ability to generate atomic models of PI to help unravel the complex problem of identifying atomic species and determining their abundances.

Although, significant progress has been made in understanding PI processes for several elements [7, 8, 9], neon ions have been the subject of relatively few PI studies, despite the fact that the presence of Ne has been confirmed in planetary nebulae [10], and that Ne is one of the most abundant elements in the Universe.

In a theoretical approach based on the close-coupling approximation by Pradhan [11, 12] the single-photon PI cross-sections for the ground states of Ne II, Ne III and Ne IV were calculated. Detailed cross-sections for a large number of states of Ne III were calculated using the non-relativistic $R$-matrix method in the $L \cdot S$ coupling approximation by Butler and Zeippen under the auspices of the Opacity Project (OP) [13] (data sets are available online [14]).

With the aid of the $R$-matrix method it has been possible, under the $L \cdot S$ coupling approximation, to construe most of the observed features and cross-section intensities in high-resolution, single-photon PI measurements of Ne II [15] and Ne IV [16] cations.

This work is concerned with the photoionization of $L$-shell electrons of $\mathrm{Ne}$, however, it is important to point out that studies of the inner shell and near $K$-edge photoionization of Ne I [17], Ne II [18] [19] are available. Isoelectronic to Be, Ne VII photoionization have also been provided [20, 21].

Ne III is isoelectronic to O I, so previous studies on the photoionization of the oxygen atom are relevant to the interpretation of the present data. Angel and Samson [22] (and references therein) identified several autoionizing states leading to $\mathrm{O}$ II and measured the relative single-photon PI cross section 
with the help of a monochromator using radiation from a synchrotron. In a calculation that included core excited states, Nahar [23] obtained the PI cross-section of the isonuclear series O I to O VII using an ab-initio $R$-matrix method. Flesh and collaborators [24] investigated the laser-induced, singlephoton ionization of excited ${ }^{1} \mathrm{D}_{2}$ state of $\mathrm{O}$ I; they found Rydberg series' below the ground state of O II. Tayal [25] calculated the single-photon PI cross section for the ground state $\mathrm{O}$ I using an $R$-matrix method. More recently, relevant parameters for states lying over the ionization limit of $\mathrm{Ne}$ III have been studied [26].

In this paper, we offer high-resolution measurements of the single-photon photoionization of the Ne III cation and an interpretation of the resulting spectra based on ab-initio coupled-channel (CC) Breit-Pauli $R$-matrix (BPRM) calculations. Energies for resonant structures and possible assignments are also provided.

\section{Theory}

We have used the close-coupling approximation in which the atomic system is represented by $(\mathrm{N}+1)$ electrons. $\mathrm{N}$ is the number of core electrons in the residual ion interacting with the $(\mathrm{N}+1)^{t h}$ electron. The $(\mathrm{N}+1)^{t h}$ electron is bound or in the continuum depending on whether its energy $(\mathrm{E})$ is negative or positive. The total wave function $\Psi_{E}$, with symmetry $J \pi$, is expressed by the expansion (for details, for example, see [27])

$$
\Psi_{E}\left(e^{-}+\text {ion }\right)=A \sum_{i} \chi_{i}(\text { ion }) \theta_{i}+\sum_{j} c_{j} \Phi_{j},
$$

where the core ion eigenfunction, $\chi_{i}$ of state $S_{i} L_{i} J_{i} \pi_{i}$, represents ground and various excited states, and the sum is over the number of considered states. The core is coupled with the wave function $\theta_{i}$ of the $(\mathrm{N}+1)^{t h}$ electron which has kinetic energy $k_{i}^{2}$ and orbital angular momentum $\ell_{i}$ in a channel labeled as $S_{i} L_{i} J_{i} \pi_{i} k_{i}^{2} \ell_{i}(S L J \pi)$. $A$ is the antisymmetrization operator. In the second sum, the $\Phi_{j}$ s are bound functions of the $(\mathrm{N}+1)$-electron system that provide for the orthogonality between the continuum and the bound electron orbitals, and account for short-range correlation. When core excitations are included in the wave function in any ab initio calculation, as in the close coupling (CC) approximation, resonances are inherently generated. Substitution of $\Psi_{E}\left(e^{-}+i o n\right)$ into the Schrödinger equation

$$
H_{N+1} \Psi_{E}=E \Psi_{E}
$$


introduces a set of coupled equations that are solved using the $R$-matrix approach. The details of the $R$-matrix method in the CC approximation can be found in e.g., [27, 28, 29, 30, 31].

Relativistic effects are included via the Breit-Pauli approximation wherein the Hamiltonian is given by

$$
\begin{aligned}
H_{N+1}^{\mathrm{BP}}= & \sum_{i=1}^{N+1}\left\{-\nabla_{i}^{2}-\frac{2 Z}{r_{i}}+\sum_{j>i}^{N+1} \frac{2}{r_{i j}}\right\}+ \\
& H_{N+1}^{\mathrm{mass}}+H_{N+1}^{\mathrm{Dar}}+H_{N+1}^{\mathrm{so}}
\end{aligned}
$$

in units of Rydberg. The Darwin relativistic terms and mass corrections are $H^{\text {Dar }}=\frac{Z \alpha^{2}}{4} \sum_{i} \nabla^{2}\left(\frac{1}{r_{i}}\right)$ and $H^{\text {mass }}=-\frac{\alpha^{2}}{4} \sum_{i} p_{i}^{4}$. The spin-orbit interaction is given by $H^{\text {so }}=Z \alpha^{2} \sum_{i} \frac{1}{r_{i}^{3}} \mathbf{l}_{\mathbf{i}} \cdot \mathbf{s}_{\mathbf{i}}$. The $R$-matrix Breit-Pauli (BPRM) approximation also includes partial two-body interaction terms, such as the ones without momentum operators [27]. In this approximation, the set of $S L \pi$ is re-coupled for the $J \pi$ levels of the $\left(\mathrm{e}^{-}+\right.$ion) system which is then followed by diagonalization of the Hamiltonian.

The solution of the BPRM approximation is a continuum wave function, $\Psi_{F}$, for an electron with positive energies ( $\mathrm{E}>0$ ), or a bound state, $\Psi_{B}$, with a negative total energy $(\mathrm{E} \leq 0)$. The complex resonant structures seen in the photoionization (PI) spectra are produced from channel couplings between continuum channels that are open $\left(k_{i}^{2}>0\right)$, and ones that are closed $\left(k_{i}^{2}<\right.$ 0 ), at electron energies $k_{i}^{2}$ corresponding to autoionizing states of the Rydberg series, $S_{i} L_{i} J_{i} \pi_{i} \nu \ell . \nu$ is the effective quantum number of the series converging to excited core thresholds.

The PI cross section $\left(\sigma_{P I}\right)$ is given by

$$
\sigma_{P I}=\frac{4 \pi^{2}}{3 c} \frac{1}{g_{i}} \omega \mathbf{S},
$$

where $g_{i}$ is the statistical weight of the bound state, $\omega$ is the incident photon energy and $\mathbf{S}$ is the generalized line strength

$$
\mathbf{S}=\left|\left\langle\Psi_{f}|| \mathbf{D}_{L}|| \Psi_{i}\right\rangle\right|^{2}=\left|\left\langle\psi_{f}\left|\sum_{j=1}^{N+1} r_{j}\right| \psi_{i}\right\rangle\right|^{2},
$$

$\Psi_{i}$ and $\Psi_{f}$ are the initial and final state wave functions, and $\mathbf{D}_{L}$ is the dipole operator in length form. 


\section{Computation}

The computations for the PI cross-sections were carried out using the BPRM package of codes [31, 32, 33]. The computation process consists of a number of stages. The first stage, STG1, is initiated with the wave function of the core ion as the initial input. The core ion wave function was obtained from atomic structure calculations using code SUPERSTRUCTURE (SS) [34, 35] which uses the Thomas-Fermi-Dirac-Amaldi potential and includes relativistic contributions from the Breit-Pauli approximation. Table 1 presents the 58 levels (the ground and 57 excited fine-structure levels) of $\mathrm{Ne}^{3+}$ included in the wave function expansion of $\mathrm{Ne}^{2+}$ obtained from the optimization of 17 configurations of the core ion. The table compares the calculated energies from SS with the observed values available in the compilation of the NIST [36], and shows a good agreement between the two.

The wave function included the set of $0 \leq \ell \leq 11$ partial waves for the interacting electron and 14 continuum functions for the $R$-matrix basis sets. The $R$-matrix boundary was chosen to be large enough $\left(\begin{array}{ll}10 & a_{o}\end{array}\right)$ to accommodate the bound orbitals. The second term of the wave function, which represents the bound state correlation functions, included $95(N+1)$-particle configurations with orbital occupancies from a minimum to a maximum number (given within the parentheses) of the orbitals $1 \mathrm{~s}(2-2), 2 \mathrm{~s}(0-2), 2 \mathrm{p}(0-6)$, $3 \mathrm{~s}(0-2), 3 \mathrm{p}(0-2), 3 \mathrm{~d}(0-2), 4 \mathrm{~s}(0-1), 4 \mathrm{p}(0-1)$.

PI cross sections are obtained considering radiation damping for all bound levels using the BPRM $R$-matrix codes [31, 32, 33]. The narrow PI resonances were delineated at a very fine energy mesh.

\section{Experiment}

The experimental setup used was located at the Advanced Light Source synchrotron at the Lawrence Berkeley National Laboratory (LBNL) and has been extensively described in previous publications. Here we offer a description which is intended to be sufficient to understand the details of this particular study. For more general aspects of the experimental method we refer the interested reader to publications by Covington et al. [15] and by Müller and collaborators [37] and also [38].

The experimental technique consisted in overlapping an ion beam and a photon beam. The ion beam was generated with an electron cyclotron resonance (ECR) ion source. For the photon beam, we used an end-line station of the synchrotron facility. 
Table 1: Levels and energies $\left(E_{t}\right)$ of core ion $\mathrm{Ne}^{3+}$ in the wave function expansion of $\mathrm{Ne}^{2+}$.

\begin{tabular}{|c|c|c|c|c|}
\hline & Level & $J_{t}$ & $\begin{array}{l}E_{t}(\mathrm{Ry}) \\
\text { NIST }\end{array}$ & $\begin{array}{l}E_{t}(\mathrm{Ry}) \\
\mathrm{SS}\end{array}$ \\
\hline 1 & $1 s^{2} 2 s^{2} 2 p^{3}\left({ }^{4} S^{\circ}\right)$ & $3 / 2$ & 0.0 & 0 . \\
\hline 2 & $1 s^{2} 2 s^{2} 2 p^{3}\left({ }^{2} D^{\circ}\right)$ & $5 / 2$ & 0.375758 & 0.4143 \\
\hline 3 & $1 s^{2} 2 s^{2} 2 p^{3}\left({ }^{2} D^{\circ}\right)$ & $3 / 2$ & 0.37616 & 0.4139 \\
\hline 4 & $1 s^{2} 2 s^{2} 2 p^{3}\left({ }^{2} P^{\circ}\right)$ & $3 / 2$ & 0.5690 & 0.5796 \\
\hline 5 & $1 s^{2} 2 s^{2} 2 p^{3}\left({ }^{2} P^{\circ}\right)$ & $1 / 2$ & 0.5689 & 0.5793 \\
\hline 6 & $1 s^{2} 2 s 2 p^{4}\left({ }^{4} P\right)$ & $5 / 2$ & 1.6755 & 1.6906 \\
\hline 7 & $1 s^{2} 2 s 2 p^{4}\left({ }^{4} P\right)$ & $3 / 2$ & 1.6811 & 1.6961 \\
\hline 8 & $1 s^{2} 2 s 2 p^{4}\left({ }^{4} P\right)$ & $1 / 2$ & 1.6840 & 1.6993 \\
\hline 9 & $1 s^{2} 2 s 2 p^{4}\left({ }^{2} D\right)$ & $5 / 2$ & 2.3153 & 2.4326 \\
\hline 10 & $1 s^{2} 2 s 2 p^{4}\left({ }^{2} D\right)$ & $3 / 2$ & 2.3155 & 2.4325 \\
\hline 11 & $1 s^{2} 2 s 2 p^{4}\left({ }^{2} S\right)$ & $1 / 2$ & 2.7304 & 2.8349 \\
\hline 12 & $1 s^{2} 2 s 2 p^{4}(2 P)$ & $3 / 2$ & 2.9163 & 3.1095 \\
\hline 13 & $1 s^{2} 2 s 2 p^{4}\left({ }^{2} P\right)$ & $1 / 2$ & 2.9228 & 3.1162 \\
\hline 14 & $1 s^{2} 2 s^{2} 2 p^{2} 3 s\left({ }^{4} P\right)$ & $1 / 2$ & 4.3622 & 4.3915 \\
\hline 15 & $1 s^{2} 2 s^{2} 2 p^{2} 3 s\left({ }^{4} P\right)$ & $3 / 2$ & 4.3657 & 4.3949 \\
\hline 16 & $1 s^{2} 2 s^{2} 2 p^{2} 3 s\left({ }^{4} P\right)$ & $5 / 2$ & 4.3710 & 4.4006 \\
\hline 17 & $1 s^{2} 2 p^{5}\left({ }^{2} P^{\circ}\right)$ & $3 / 2$ & 4.4188 & 4.6890 \\
\hline 18 & $1 s^{2} 2 p^{5}\left({ }^{2} P^{\circ}\right)$ & $1 / 2$ & 4.4275 & 4.6986 \\
\hline 19 & $1 s^{2} 2 s^{2} 2 p^{2} 3 s\left({ }^{2} P\right)$ & $1 / 2$ & 4.4516 & 4.4976 \\
\hline 20 & $1 s^{2} 2 s^{2} 2 p^{2} 3 s\left({ }^{2} P\right)$ & $3 / 2$ & 4.4579 & 4.5043 \\
\hline 21 & $1 s^{2} 2 s^{2} 2 p^{2} 3 p\left({ }^{2} S^{\circ}\right)$ & $1 / 2$ & & 4.7018 \\
\hline 22 & $1 s^{2} 2 s^{2} 2 p^{2} 3 s\left({ }^{2} D\right)$ & $5 / 2$ & 4.6628 & 4.7094 \\
\hline 23 & $1 s^{2} 2 s^{2} 2 p^{2} 3 s\left({ }^{2} D\right)$ & $3 / 2$ & 4.6630 & 4.7094 \\
\hline 24 & $1 s^{2} 2 s^{2} 2 p^{2} 3 p\left({ }^{4} D^{\circ}\right)$ & $1 / 2$ & 4.7478 & 4.7572 \\
\hline 25 & $1 s^{2} 2 s^{2} 2 p^{2} 3 p\left({ }^{4} D^{\circ}\right)$ & $3 / 2$ & 4.7498 & 4.7592 \\
\hline 26 & $1 s^{2} 2 s^{2} 2 p^{2} 3 p\left({ }^{4} D^{\circ}\right)$ & $5 / 2$ & 4.7530 & 4.7626 \\
\hline 27 & $1 s^{2} 2 s^{2} 2 p^{2} 3 p\left({ }^{4} D^{\circ}\right)$ & $7 / 2$ & 4.7573 & 4.7674 \\
\hline 28 & $1 s^{2} 2 s^{2} 2 p^{2} 3 p\left({ }^{4} P^{\circ}\right)$ & $1 / 2$ & 4.7795 & 4.7965 \\
\hline 29 & $1 s^{2} 2 s^{2} 2 p^{2} 3 p\left({ }^{4} P^{\circ}\right)$ & $3 / 2$ & 4.7812 & 4.7984 \\
\hline 30 & $1 s^{2} 2 s^{2} 2 p^{2} 3 p\left({ }^{4} P^{\circ}\right)$ & $5 / 2$ & 4.7844 & 4.8018 \\
\hline 31 & $1 s^{2} 2 s^{2} 2 p^{2} 3 p\left({ }^{2} D^{\circ}\right)$ & $3 / 2$ & 4.8367 & 4.8643 \\
\hline 32 & $1 s^{2} 2 s^{2} 2 p^{2} 3 p\left({ }^{2} D^{\circ}\right)$ & $5 / 2$ & 4.8569 & 4.8710 \\
\hline 33 & $1 s^{2} 2 s^{2} 2 p^{2} 3 p\left({ }^{4} S^{\circ}\right)$ & $3 / 2$ & 4.8569 & 4.8730 \\
\hline 34 & $1 s^{2} 2 s 2 p^{3} 3 s\left({ }^{6} S^{\circ}\right)$ & $5 / 2$ & 4.9072 & 5.0673 \\
\hline 35 & $1 s^{2} 2 s^{2} 2 p^{2} 3 p\left({ }^{2} P^{\circ}\right)$ & $1 / 2$ & & 4.9367 \\
\hline 36 & $1 s^{2} 2 s^{2} 2 p^{2} 3 p\left({ }^{2} P^{\circ}\right)$ & $3 / 2$ & & 4.9372 \\
\hline 37 & $1 s^{2} 2 s^{2} 2 p^{2} 3 s\left({ }^{2} S\right)$ & $1 / 2$ & 5.0301 & 5.1169 \\
\hline 38 & $1 s^{2} 2 s^{2} 2 p^{2} 3 p\left({ }^{2} F^{\circ}\right)$ & $5 / 2$ & 5.0602 & 5.0983 \\
\hline 39 & $1 s^{2} 2 s^{2} 2 p^{2} 3 p\left({ }^{2} F^{\circ}\right)$ & $7 / 2$ & 5.0614 & 5.0997 \\
\hline 40 & $1 s^{2} 2 s^{2} 2 p^{2} 3 p\left({ }^{2} D^{\circ}\right)$ & $5 / 2$ & 5.1133 & 5.1863 \\
\hline 41 & $1 s^{2} 2 s^{2} 2 p^{2} 3 p\left({ }^{2} D^{\circ}\right)$ & $3 / 2$ & 5.1143 & 5.1868 \\
\hline 42 & $1 s^{2} 2 s^{2} 2 p^{2} 3 p\left({ }^{2} P^{\circ}\right)$ & $1 / 2$ & & 5.2463 \\
\hline 43 & $1 s^{2} 2 s^{2} 2 p^{2} 3 p\left({ }^{2} P^{\circ}\right)$ & $3 / 2$ & & 5.2495 \\
\hline 44 & $1 s^{2} 2 s^{2} 2 p^{2} 3 d\left({ }^{2} P\right)$ & $3 / 2$ & 5.2511 & 5.3040 \\
\hline 45 & $1 s^{2} 2 s^{2} 2 p^{2} 3 d\left({ }^{4} F\right)$ & $3 / 2$ & & 5.2679 \\
\hline 46 & $1 s^{2} 2 s^{2} 2 p^{2} 3 d\left({ }^{4} F\right)$ & $5 / 2$ & & 5.2697 \\
\hline 47 & $1 s^{2} 2 s^{2} 2 p^{2} 3 d\left({ }^{4} F\right)$ & $7 / 2$ & & 5.2725 \\
\hline 48 & $1 s^{2} 2 s^{2} 2 p^{2} 3 d\left({ }^{4} F\right)$ & $9 / 2$ & & 5.2761 \\
\hline 49 & $1 s^{2} 2 s^{2} 2 p^{2} 3 d\left(^{2} P\right)$ & $1 / 2$ & 5.2547 & 5.3116 \\
\hline 50 & $1 s^{2} 2 s^{2} 2 p^{2} 3 d\left({ }^{2} D\right)$ & $3 / 2$ & 5.2599 & 5.4149 \\
\hline 51 & $1 s^{2} 2 s^{2} 2 p^{2} 3 d\left({ }^{2} D\right)$ & $5 / 2$ & 5.2599 & 5.4168 \\
\hline 52 & $1 s^{2} 2 s^{2} 2 p^{2} 3 d\left({ }^{4} P\right)$ & $5 / 2$ & 5.2790 & 5.3275 \\
\hline 53 & $1 s^{2} 2 s^{2} 2 p^{2} 3 d\left({ }^{4} P\right)$ & $3 / 2$ & 5.2819 & 5.3300 \\
\hline 54 & $1 s^{2} 2 s^{2} 2 p^{2} 3 d\left({ }^{4} P\right)$ & $1 / 2$ & 5.2830 & 5.3313 \\
\hline 55 & $1 s^{2} 2 s^{2} 2 p^{2} 3 d\left({ }^{4} D\right)$ & $1 / 2$ & & 5.3059 \\
\hline 56 & $1 s^{2} 2 s^{2} 2 p^{2} 3 d\left({ }^{4} D\right)$ & $5 / 2$ & & 5.3090 \\
\hline 57 & $1 s^{2} 2 s^{2} 2 p^{2} 3 d\left({ }^{4} D\right)$ & $3 / 2$ & & 5.3092 \\
\hline 58 & $1 s^{2} 2 s^{2} 2 p^{2} 3 d\left({ }^{4} D\right)$ & $7 / 2$ & & 5.3109 \\
\hline
\end{tabular}


The experiment was designed to measure photoionization cross sections. In this study, we report on the cross-section of the following reaction,

$$
\mathrm{Ne}^{2+}+h \nu \longrightarrow \mathrm{Ne}^{3+}+e^{-},
$$

in which the resulting $\mathrm{Ne}^{3+}$ (Ne IV) ions, or photo-ions, were counted as a function of the photon energy and relevant parameters of the ion beam and the photon beam together with the spatial overlap of the two beams monitored.

To produce the ion beam of $\mathrm{Ne}^{2+}$ (Ne III), a mixture of $\mathrm{Ne}$ and $A r$ gases was injected into the all-permanent magnets ECR ion source. A $60^{\circ}$-sector analyzing magnet was subsequently used to select the $\mathrm{Ne}^{2+}$ cations which had a kinetic energy of $12 \mathrm{keV}$. With the help of an electrostatic Einzel lens, together with two sets of steering plates and a set of slits, the ion beam was focused and collimated. Subsequently, an electrostatic $90^{\circ}$ sphericalsector analyzer was used to merge the ion beam with the counter-propagating photon beam.

The key section of this experiment was a voltage-biased metal-mesh cylinder where the $\mathrm{Ne}^{3+}$-photoions produced inside coupled with a different electrostatic potential than the potential experienced by ions produced outside. This metal-mesh cylinder section is defined as the interaction region (IR). The photoions generated inside this IR were separated from those produced outside the IR via a $45^{\circ}$ dipole analyzing magnet located downstream (as measured by the direction of ion beam travel) from the IR.

To produce the photon beam, the end line of the synchrotron was equipped with an undulator located inside the ring. The period of the undulator was $10 \mathrm{~cm}$. The synchrotron was operated at $1.9 \mathrm{GeV}$ under a constant electron current of $500 \mathrm{~mA}$. The photon beam was directed onto a spherical grating with controls to scan the photon beam energy and to select the energy resolution. The resulting photon beam had a spatial width less than $1.5 \mathrm{~mm}$, a maximum divergence of $0.06^{\circ}$ and an energy resolution of $8.2 \mathrm{meV}$. This resolution was estimated from the widths of the resulting resonant peaks in the spectra.

To calibrate the photon energy, an ionization gas-cell was implemented on a side branch of the photon beam line. In this cell, $\mathrm{He}$ or $\mathrm{Kr}$ gases were irradiated in the approximate energy range of $18.6 \mathrm{eV}$ to $92.4 \mathrm{eV}$. For reference values, we used the data by King et al. [39] and Domke and collaborators [40]. The Doppler shift associated with the counter-propagating 
beams was also taken into consideration. An energy uncertainty of $\pm 10 \mathrm{meV}$ was estimated as a result of this particular energy calibration procedure.

In the experiment, the resulting $\mathrm{Ne}^{3+}$ photoions were counted as a function of the photon energy. This procedure resulted in an energy spectrum of the relative intensity of the $\mathrm{Ne}^{3+}$ signal that was later normalized to absolute cross-section measurements.

To measure the absolute cross-section, exact knowledge of the interaction region dimensions and of the overlap of the two beams was required. To this end, two-dimensional beam profiles of the ion beam $I^{+}(x, y)$ and the photon beam $I^{\gamma}(x, y)$ were measured. Three beam profilers were used to sample the form factor $F(z)$ according to

$$
F_{i}(z)=\frac{\iint I^{+}(x, y) I^{\gamma}(x, y) d x d y}{\iint I^{+}(x, y) d x d y \iint I^{\gamma}(x, y) d x d y},
$$

where $z$ is the reference axis assigned to the propagation direction of the ion beam. $F(z)$ was measured at three positions; at the entrance, in the center, and at the exit of the IR. These three values, $F_{i}(z)$, were used to derive $F(z)$ by interpolation along the total IR length and derive, by integration over $z$, the spatial overlap of the photon and ion beams along the common IR path [15].

The single-photon photoionization cross-section, $\sigma$, for $\mathrm{Ne}^{2+}$ was computed from

$$
\sigma=\frac{R q e^{2} v_{i} \epsilon}{I^{+} I^{\gamma} \int F(z) d z},
$$

where $R$ is the photoion count rate, $q=2$ is the charge state of $\mathrm{Ne}^{2+}$, $e=1.6 \times 10^{-19} \mathrm{C}, v_{i}$ is the ion beam velocity in $\mathrm{cm} \cdot \mathrm{s}^{-1}, \epsilon$ is the responsivity of the photo-diode (electrons per photon), $I^{+}$is the ion beam current (A), and $I^{\gamma}$ is the photo-diode current $(\mathrm{A})$.

The $\mathrm{Ne}^{2+}$ ion beam was measured in an extended Faraday cup. The resulting $\mathrm{Ne}^{3+}$ photoions were counted on a single-channel detector located aft of the dipole analyzing magnet whose magnetic field intensity was adjusted so that only photoions generated inside the IR were collected. A background subtraction of the $\mathrm{Ne}^{3+}$ signal was performed by cutting the photon beam with a chopper wheel. In this manner, $\mathrm{Ne}^{3+}$ ions produced by collisions with residual gas were eliminated. 
The most important sources of systematic error in $\sigma$ in Eq. 8 came from the beams-overlap integral, the beams profile measurements, and the photodiode responsivity function. Other contributions to the total systematic error are listed in Covington et al. [15]. All sources of systematic error combine to a total uncertainty of $20 \%$.

The energy spectrum of the relative intensity of $\mathrm{Ne}^{3+}$ was normalized to absolute cross-section measurements that were performed at specific energy values of the spectrum at which no resonant peaks were found. A normalization function was derived for these energy values by taking the ratio of the cross-section to the relative intensity spectrum.

The spectra shown in Fig. 1 and Fig. 2] were measured at 5 to $10 \mathrm{eV}$-wide photon energy intervals. Each interval overlapped its neighboring interval by $1.0 \mathrm{eV}$. All the individual parts of the spectra were later combined by matching peaks between adjacent intervals. An estimation of the maximum possible photon energy uncertainty caused by this procedure was $\pm 8 \mathrm{meV}$. Therefore, the overall energy uncertainty propagated by both the gas-cell energy calibration and the data reduction procedure is $\pm 13 \mathrm{meV}$.

\section{Results}

The measured cross-section is shown in Figs. 11 and 2, Results of the calculations for the single-photon PI cross-section of Ne III are presented in Figs. 3, 4] and 5,

The spectrum consists of several resonant peaks superimposed on a somewhat tenuous background. Most of the resonant peaks are attributed to Rydberg series whose energies are given by

$$
E_{n}=E_{\infty}-\frac{z^{2} R}{(n-\delta)^{2}},
$$

where $E_{n}$ are the resonant energies, $E_{\infty}$ is the energy limit, $z$ is the charge state of ion core (in this case 3 ), $R=13.606 \mathrm{eV}, n$ is the principal quantum number and $\delta$ is the quantum defect.

The energy limits $E_{\infty}$ in the spectra of Figs. 1 and 2 and in Tables 2, 3, 4 and 5] are computed from the ionization energy of the ground state (indicated by IE in Fig. 1), minus the excitation energy of the initial $\mathrm{Ne}^{2+}$ state, plus the excitation energy of the final $\mathrm{Ne}^{3+}$ state. Using this derivation for $E_{\infty}$, series were identified. As reference values for the ionization energies, excitation energies and term splittings, we used tabulated NIST values [36]. 
In Figs. 1 and 2, resonant energies $E_{n}$ are indicated by vertical lines grouped by an inclined or horizontal line according to the series to which they are assigned. Roman numbers are used to identify the series in the figures and in tables 2, 3, 4 and 5. In each of the series in Figs. 1 and 2, the last vertical line of the group corresponds to the series limit $E_{\infty}$.

Below the ionization energy, one small series was identified as originating from the excited state $\mathrm{Ne}^{2+},\left(2 s^{2} 2 p^{4}\right)^{1} S_{0}$. This series is labelled by I in Fig. 1 and in Table 2. This series converges to $\mathrm{Ne}^{3+}\left(2 s^{2} 2 p^{3}\right)^{2} P_{1 / 2,3 / 2}^{\circ}$. This doublet $P$ term is split by $1 \mathrm{meV}$ and is unresolved in this experiment.

Series labelled by II and III originate from the first excited state of $\mathrm{Ne}^{2+}$ $\left(2 s^{2} 2 p^{4}\right)^{1} D_{2}$ and are superimposed over one another. They converge to the doublets $\mathrm{Ne}^{3+}\left(2 s^{2} 2 p^{3}\right)^{2} D_{5 / 2,3 / 2}^{\circ}$ and ${ }^{2} P_{1 / 2,3 / 2}^{\circ}$. The energy split of these doublets is $6 \mathrm{meV}$ and $1 \mathrm{meV}$ respectively and could not be resolved by the experiment. The only resonance that seems "doubled-peaked" is the first and largest structure of the spectrum and may be attributed to the ${ }^{2} D_{5 / 2,3 / 2}$ splitting. Auto-ionizing states of $\mathrm{Ne}^{2+}$ that may converge to these final states of $\mathrm{Ne}^{3+}$ are $2 s^{2} 2 p^{3}\left({ }^{2} D^{\circ}\right) n s{ }^{1} D_{2}^{\circ} ; 2 s^{2} 2 p^{3}\left({ }^{2} D^{\circ}\right) n d{ }^{1} F_{3}^{\circ},{ }^{1} D_{2}^{\circ},{ }^{1} P_{1}^{\circ}$; $2 s^{2} 2 p^{3}\left({ }^{2} P^{\circ}\right) n s^{1} P_{1}^{\circ}$ and $2 s^{2} 2 p^{3}\left({ }^{2} P^{\circ}\right) n d{ }^{1} D_{2}[41]$.

In Fig. 1, series labelled by IV, V and VI are intermingled. These series are identified as originating from the ground state $\mathrm{Ne}^{2+}\left(2 s^{2} 2 p^{4}\right)^{3} P_{2,1,0}$. Their final state seems to be the first excited state of $\mathrm{Ne}^{3+}\left(2 s^{2} 2 p^{3}\right)^{2} D_{5 / 2,3 / 2}^{\circ}$. The energy splitting of the final state term is $6 \mathrm{meV}$ and may not be resolved by the experiment. Since these series are overlapped, their assignment as well as the peak energy values are tentative. The series that converge to this final state are $2 s^{2} 2 p^{3}\left({ }^{2} D^{\circ}\right) n s^{3} D^{\circ}$ and $2 s^{2} 2 p^{3}\left({ }^{2} D^{\circ}\right) n d^{3} S^{\circ},{ }^{3} D$ [25]. We offer possible assignments to the series labeled by IV, V and VI of Fig. 1 and Table 3 .

Below the ionization threshold of $\mathrm{Ne}^{2+}\left(2 s^{2} 2 p^{4}\right)^{1} D_{2}(66.623 \mathrm{eV})$, the small background may be caused by the presence of excited $\mathrm{Ne}^{2+}\left(2 s^{2} 2 p^{4}\right)^{1} S_{0}$ in the ion beam, and has been identified as series I. The small magnitude of the signal is due to the fact that transitions of the $\mathrm{Ne}^{2+}$ singlet states ${ }^{1} D_{2}$ and ${ }^{1} S_{0}$ to $\mathrm{Ne}^{3+}\left(2 s^{2} 2 p^{3}\right)^{4} S_{3 / 2}^{\circ}$ are spin-forbidden [42].

In the energy range of approximately 68 to $71 \mathrm{eV}$ of Fig. 1, three Rydberg series labelled by VII have been identified. The first discernible peak of these series corresponds to $n=7$. The series can be clearly followed up to $\mathrm{n}=15$. The largest and clearer series corresponds to the ground state $\mathrm{Ne}^{2+}$ $\left(2 s^{2} 2 p^{4}\right)^{3} P_{2}$ converging to the doublet $\left(2 s^{2} 2 p^{3}\right)^{2} D^{\circ} \mathrm{J}=3 / 2$ and $5 / 2$. According to the NIST tables, this doublet is split by $1 \mathrm{meV}$ and therefore cannot 
be resolved by this experiment. The second series in this energy range corresponds to the next member of the ground state triplet of $\mathrm{Ne}^{2+}\left(2 s^{2} 2 p^{4}\right)^{3} P_{1}$ and, with a lower intensity but still somewhat resolved, it appears the last member of the ground state triplet $\mathrm{Ne}^{2+}\left(2 s^{2} 2 p^{4}\right)^{3} P_{0}$. Gaussian-fit centers for this series are tabulated in Table 4. Autoionizing states that may converge to these series are $2 s^{2} 2 p^{3}\left({ }^{2} P^{\circ}\right) n s^{3} D^{\circ}$ and $2 s^{2} 2 p^{3}\left({ }^{2} P^{\circ}\right) n d^{3} D^{\circ},{ }^{3} P^{\circ}$.

Peaks around and about $73 \mathrm{eV}$ of Fig. 2 have not been associated with a Rydberg series. According to our calculations, these peaks are resolved when the initial state of $\mathrm{Ne}^{2+}$ corresponds to $\left(2 s^{2} 2 p^{4}\right)^{1} D_{2}$. Gaussian-fit centers for these resonances are given in the Fig. 2 caption.

In Fig. 2, centered approximately at $81 \mathrm{eV}$, the series labeled by VIII originating from the excited state $\mathrm{Ne}^{2+}\left(2 s^{2} 2 p^{5}\right)^{3} P_{2}^{\circ}$, converges to the first $s$ excited state of $\mathrm{Ne}^{3+}\left(2 s 2 p^{4}\right)^{4} P_{5 / 2,3 / 2,1 / 2}$. They all appear as wide peaks in the experiment even when all final and initial states are fully separated. In Table 5, Gaussian-fit centers for this series are presented.

In Fig. 2, resonant structures centered around 87.5 are associated with the final state $\mathrm{Ne}^{3+}\left(2 s 2 p^{4}\right)^{2} D_{5 / 2}$ at $91.721 \mathrm{eV}$ and the initial state $\mathrm{Ne}^{2+}$ $\left(2 s^{2} 2 p^{4}\right)^{1} D_{2}$. This series is labelled as series IX. It seems there exists two intermediate states with $\delta=0.40$ and 0.48 . It was possible to distinguish the peaks at lower energies, but at higher energies both series merge. See Table 5

The Rydberg series of resonances belonging to core ion excitations are relatively well separated, at about $0.37 \mathrm{Ry}$ for ${ }^{2} D_{5 / 2,3 / 2}^{\circ}, 0.57 \mathrm{Ry}$ for ${ }^{2} P_{3 / 2,1 / 2}^{\circ}$ of the ground state configurations, and about 1.68 Ry for ${ }^{4} P_{5 / 2,3 / 2,1 / 2}$ and, 2.3 Ry for ${ }^{2} D_{5 / 2,3 / 2}$ levels (see Table 1). Hence, mixing of resonances belonging to different states is minimal and represents represents one main reason for their accurate identification.

The matching of resonances between the measurement and the theory is found to be excellent for Ne III (Figs. 3, 4, 5). Some differences are expected, particularly in the peaks of the resonances. The peak of the measured resonance may depend on: (i) the interference of the autoionizing series of resonances, (ii) the relative populations of the ground and excited levels, and (iii) changes in the physical conditions, such as the density and temperature of the experimental ion beam. While condition (iii) has been neglected, the precise values of population of the levels remain undetermined. However, the agreement between the measurement and theory (Figs. 3, 4, 5) determines the existence of levels of $2 s^{2} 2 p^{4}\left({ }^{3} P_{0,1,2},{ }^{1} D_{2},{ }^{1} S_{0}\right)$, and $2 s 2 p^{5}\left({ }^{1} P_{1}^{\circ},{ }^{3} P_{0,1,2}^{\circ}\right)$ in 
the ion beam and most probably to the statistical distribution.

\subsection{Comparison with experiment}

Detailed comparisons between the measured and predicted spectrum of $\sigma_{P I}$ is presented in Figs. 3, 4 and 5. A remarkable aspect of this experiment, apart from its high resolution, is the close match of the measured spectrum against theory. Resonances appear clearly and allow direct assignments to the theoretically predicted resonances. This makes the manipulation of data through convolution redundant.

The measured spectrum has features, integrated from eight low-lying levels, five from the ground configuration $2 s^{2} 2 p^{4}\left({ }^{3} P_{2,1,0},{ }^{1} D_{2},{ }^{1} S_{0}\right)$ (Fig. 3) and four from the next excited configuration $2 s 2 p^{5}\left({ }^{3} P_{0,1,2}^{\circ},{ }^{1} P_{1}^{\circ}\right)$ (Fig. 44). A number of these levels yield similar features at about the same photon energies. To illustrate the combined features appearing in the observed PI spectrum, the spectrum is divided into three plots: Figs. 3, 4 and 5. Most of the prominent resonant structures in the observed spectrum have been marked by arrows in all panels presenting cross-sections of the nine levels in order to properly identify the relevant structures.

Fig. 3 a compares the observed spectrum with calculated spectra originating from the three levels ${ }^{3} P_{2}$ and ${ }^{3} P_{1,0}$ of the ground state ${ }^{3} P$. Most of the structures below $68 \mathrm{eV}$ in the observed spectrum are due to Rydberg series of resonances belonging to $2 s^{2} 2 p^{3}\left({ }^{2} D_{5 / 2,3 / 2}^{2}\right) n(s, d)$ converging to the residual ion state ${ }^{2} D^{\circ}$ (lying about $5 \mathrm{eV}$ above the ionization threshold). Comparison with theory shows that most of the observed features come from these three levels. The experimental parameters were set largely in an effort to observe these features. In the lower-energy region, the observed features up to a photon energy of about $71 \mathrm{eV}$ match with those of the ground state beyond which (up to $75 \mathrm{eV}$ ) some features are missing. Features in the middle-energy region of about 76 to $86 \mathrm{eV}$ are identified in the predicted cross-sections of the ground state. However, the higher-energy region features in the energy range of 87 to $91 \mathrm{eV}$ (except the one at $90 \mathrm{eV}$ ), are also missing.

Fig. 4 shows the comparison of the observed spectrum with the calculated spectra originating from the other two terms ${ }^{1} D_{2}$ and ${ }^{1} S_{0}$ of the ground configuration. While resonances similar to those of the state ${ }^{3} P$ appear for the ${ }^{1} D_{2}$ level (and partially for the ${ }^{1} S_{0}$ level), and thus contribute to the combined spectrum, missing features in the energy region of 71 to $75 \mathrm{eV}$ of $\sigma_{P I}$ can now be seen in the levels ${ }^{1} D_{2}$ and ${ }^{1} S_{0}$. Those in the energy region of 71 to $73 \mathrm{eV}$ originate from the $\sigma_{P I}$ of ${ }^{1} D_{2}$ and the one at $74.3 \mathrm{eV}$ 
originates from the $\sigma_{P I}$ of ${ }^{1} S_{0}$. They are, however, slightly shifted to the left by the configuration interaction in the theoretical calculations. The missing structures in the high-energy region of 87 to $91 \mathrm{eV}$ also appear in the $\sigma_{P I}$ for ${ }^{1} D_{2}$ and ${ }^{1} S_{0}$.

Fig. 5 compares the observed spectrum with the calculated spectra originating from the four excited levels, $2 s 2 p^{5}\left({ }^{1} P_{1}^{\circ},{ }^{3} P_{0,1,2}^{\circ}\right)$, all of which are higherlying levels compared to those of the ground configuration. Nonetheless they still contribute to the observed spectrum, particularly around the energy interval of 64 to $67 \mathrm{eV}$. The term ${ }^{1} P_{1}^{\circ}$ contributes significantly to the background in the region 68 to $72.5 \mathrm{eV}$ of the observed spectrum.

\section{Conclusions}

In this study, the single-photon photoionization cross-section of Ne III leading to Ne IV has been measured in the energy range from below the ground state ionization energy up to $92 \mathrm{eV}$. The experimental results have been compared with predictions calculated via the relativistic $R$-matrix BreitPauli (BPRM) approximation The photon energy resolution of the experiment was $8.2 \mathrm{meV}$. For the theoretical calculations, a close-coupling wave function expansion including 58 fine-structure levels of the residual ion $\mathrm{Ne}$ IV was included.

The spectrum exhibits several peaks, most of which are due to Rydberg series resonances. Identifications and energies for most of these resonances are given. It was possible to identify features originating from lower-lying states of Ne III (of the electronic configuration $\left(2 s^{2} 2 p^{4}\right)$, terms ${ }^{3} P_{210},{ }^{1} D_{2}$, and $\left.{ }^{1} S_{0}\right)$, and also from the $\left(2 s 2 p^{5}\right)$ term ${ }^{3} P_{210}^{\circ}$. In the theoretical calculations, the next excited state of Ne III, $\left(2 s 2 p^{5}\right)$ term ${ }^{1} P_{1}^{\circ}$, was also included, and some peaks associated with this state were clearly identified. As for the core final states identified in the experiment, it was possible to realize all of the first five lower-lying terms of $\mathrm{Ne}^{3+}$. The present study also found negligible contributions from relativistic effects at the high-energy range of the study.

We hope the present study serves as a benchmark for future photoionization applications and theoretical studies of neon ions and oxygen isoelectronic ions.

\section{Acknowledgements}

The Advanced Light Source is supported by the Director, Office of Science, Office of Basic Energy Sciences, of the US Department of Energy un- 
der Contract No. DE-AC02-05CH11231. A. M. C. acknowledges support through Cooperative Agreement doe-na0002075. S. N. N. acknowledges partial support from NSF AST-1312441 and DOE de-sc0012331 and that the computational work was carried out at the Ohio Supercomputer Center in Columbus Ohio. G. H. acknowledges UNAM PAPIIT IN-109-317. 


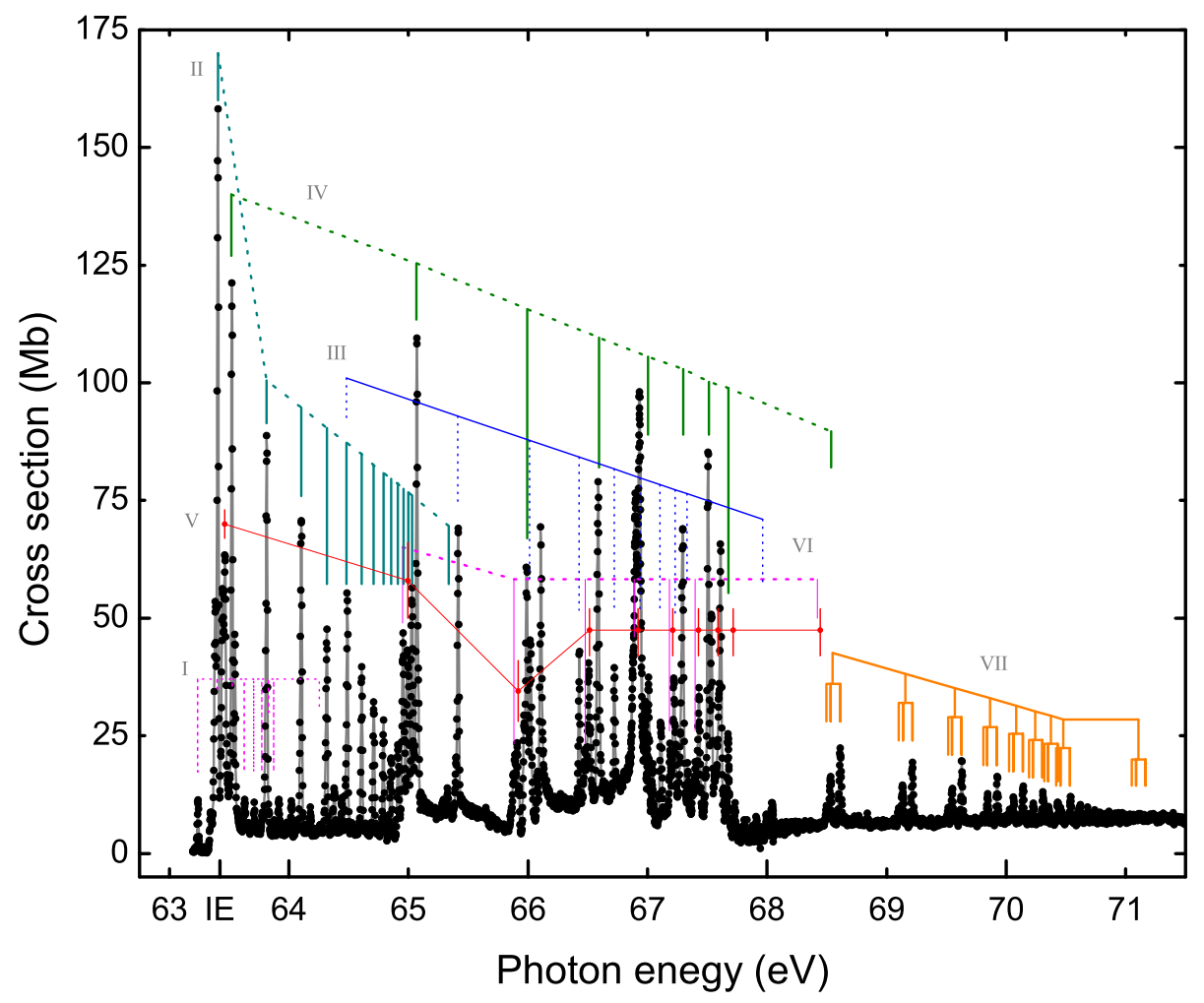

Figure 1: (Color online) Single-photon photoionization cross-section for $\mathrm{Ne}^{2+}+h \nu \rightarrow$ $\mathrm{Ne}^{3+}+e^{-}$. For clarity, the rest of the measured spectrum is shown in Fig. 2. The identified Rydberg series are grouped with inclined lines that intersect with vertical lines. The vertical lines indicate the positions of the resonances $\left(E_{n}\right)$ in the spectrum. The last vertical line of each group corresponds to the limit $E_{\infty}$. Each group of lines is identified with a Roman number in Tables 2, 3 and 4. Label IE indicates the position of the ground state ionization energy. 


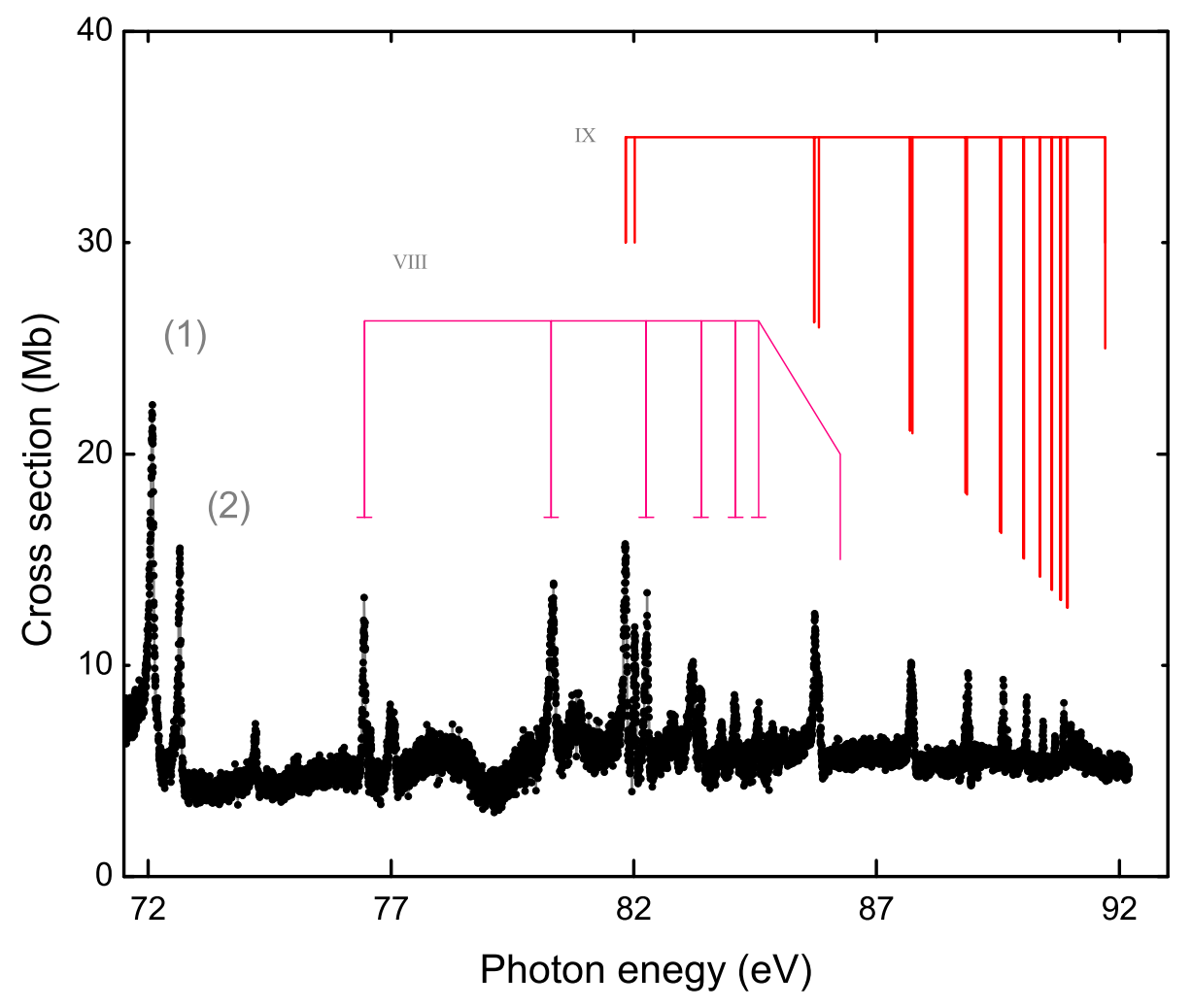

Figure 2: (Color online) Single-photon photoionization cross-section for $\mathrm{Ne}^{2+}+h \nu \rightarrow$ $\mathrm{Ne}^{3+}+e^{-}$. This figure is a continuation of Fig. 1. Note the vertical scale is different. As in Fig. 1, identified Rydberg series are grouped with horizontal lines that intersect with vertical lines. The vertical lines indicate the resonance positions $\left(E_{n}\right)$ in the spectrum. The last vertical line of each group corresponds to the limit $E_{\infty}$. Groups of lines are identified with Roman numbers in Tables 4 and 5. The unidentified peak labelled by (1) has a Gaussian center at $72.074 \mathrm{eV}, I=0.09 \mathrm{eV} \cdot \mathrm{Mb}$ and $\omega=63 \mathrm{meV}$. The peak labelled by (2) has a Gaussian center at $72.646 \mathrm{eV}, I=0.45 \mathrm{eV} \cdot M b$ and $\omega=42 \mathrm{meV}$. 


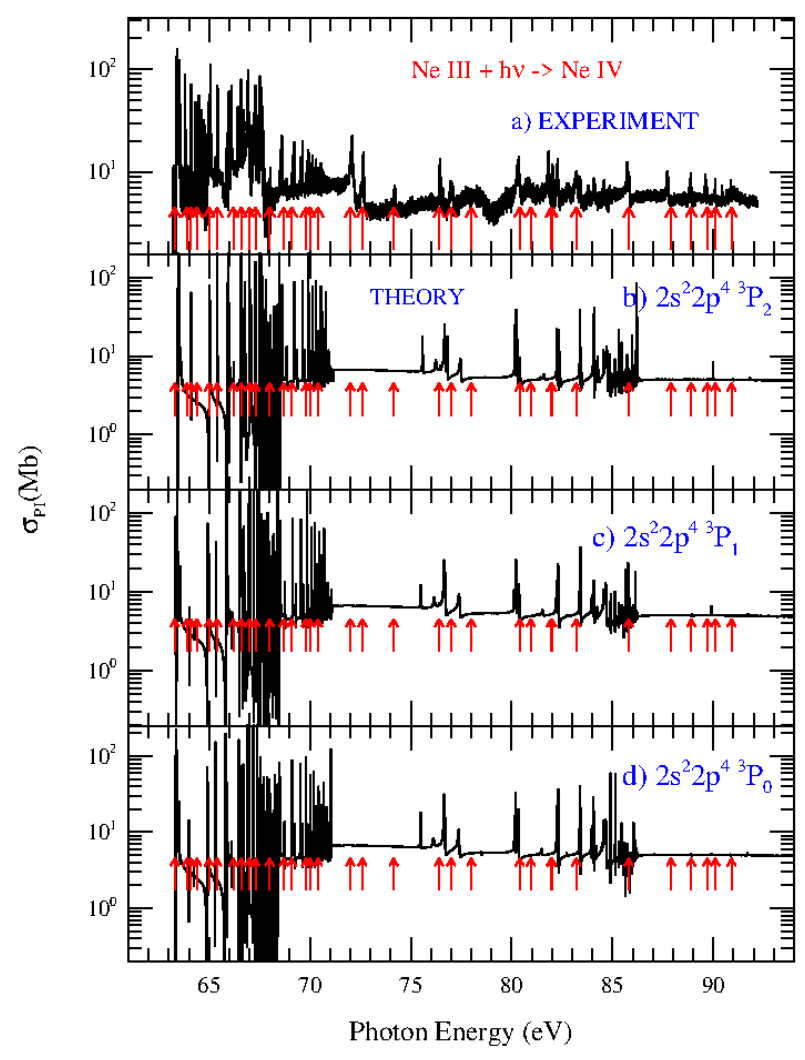

Figure 3: (Color online) Comparison between the measured and the calculated singlephoton photoionization cross-sections of $\mathrm{Ne}^{2+}$. Note the vertical logarithmic scale. The top panel corresponds to the experimental spectrum. The lower panels show the theoretical results for the indicated initial states of $\mathrm{Ne}^{2+}$. Arrows indicate the positions of some resonances to assist in their assignments. 


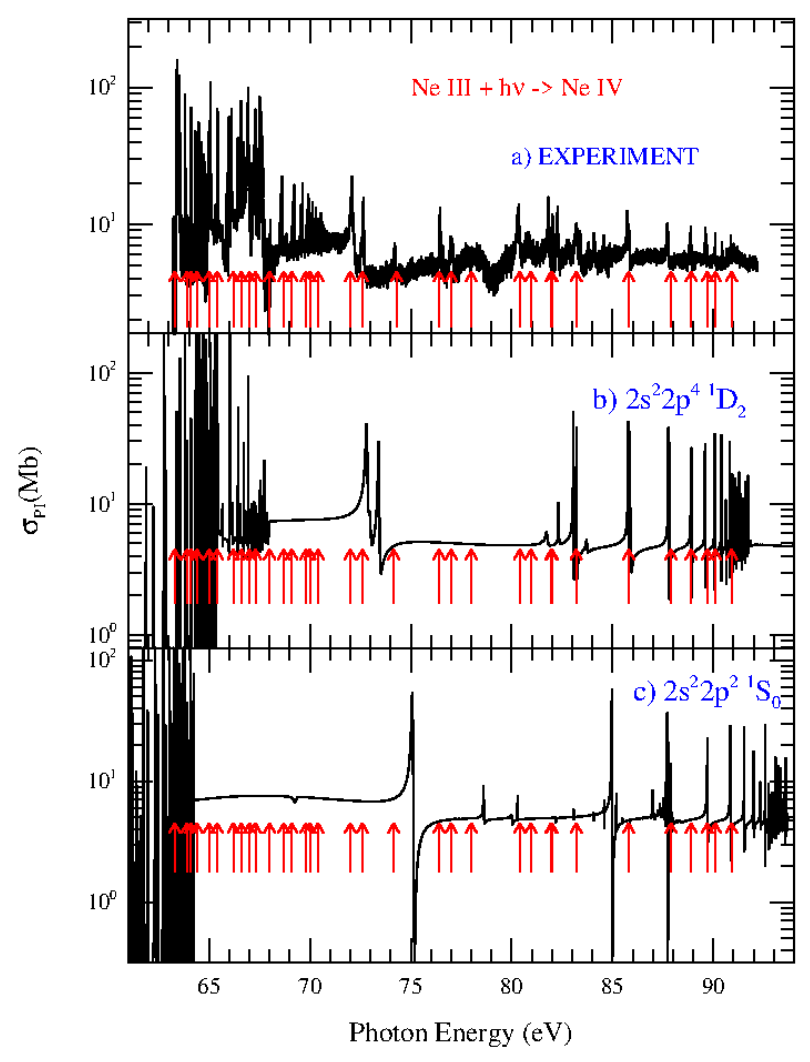

Figure 4: (Color online) Comparison between the measured and the calculated singlephoton photoionization cross-sections of $\mathrm{Ne}^{2+}$. Note the vertical logarithmic scale. The top panel corresponds to the experimental spectrum. The lower panels show the theoretical results for the indicated initial states of $\mathrm{Ne}^{2+}$. Arrows indicate the positions of some resonances to assist in their assignments. 


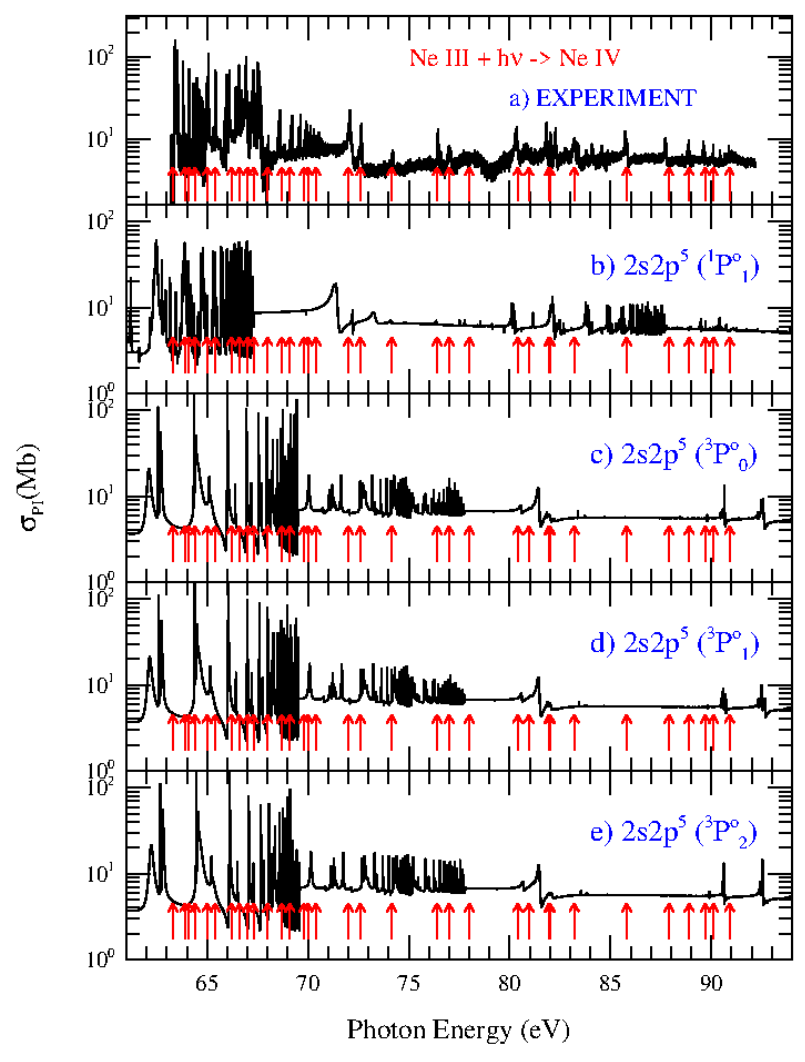

Figure 5: (Color online) Comparison between the measured and the calculated singlephoton photoionization cross sections of $\mathrm{Ne}^{2+}$. Note the vertical logarithmic scale. The top panel corresponds to the experimental spectrum. The lower panels show the theoretical results for the indicated initial states of $\mathrm{Ne}^{2+}$. Arrows indicate the positions of some resonances to assist in their assignments. 


\begin{tabular}{|c|c|c|c|}
\hline$n$ & $\begin{array}{c}E_{n} \\
(\mathrm{eV})\end{array}$ & $\begin{array}{c}I \\
(e V \cdot M b)\end{array}$ & $\begin{array}{c}\omega \\
(m e V)\end{array}$ \\
\hline \multicolumn{4}{|c|}{$\begin{array}{c}N e^{2+}\left(2 s^{2} 2 p^{4}\right)^{1} S_{0} \rightarrow N e^{3+}\left(2 s^{2} 2 p^{3}\right)^{2} P_{1 / 2,3 / 2} \\
\delta=0.02\end{array}$} \\
\hline 11 & 63.238 & 0.14 & 11.0 \\
\hline 12 & - & - & - \\
\hline 13 & - & - & - \\
\hline 14 & 63.629 & 0.08 & 12.0 \\
\hline 15 & 63.711 & 0.04 & 7.0 \\
\hline 16 & $(63.778)$ & $(0.02)$ & - \\
\hline 17 & 63.877 & 0.02 & 8.0 \\
\hline$\vdots$ & - & - & - \\
\hline$\infty$ & 64.253 & - & - \\
\hline \multicolumn{4}{|c|}{$\begin{aligned} N e^{2+}\left(2 s^{2} 2 p^{4}\right)^{1} D_{2} & \rightarrow N e^{3+}\left(2 s^{2} 2 p^{3}\right)^{2} D_{5 / 2} \\
\delta & =0.02\end{aligned}$} \\
\hline 8 & 63.406 & 1.52 & 9.6 \\
\hline 9 & 63.812 & 1.01 & 11.0 \\
\hline 10 & 64.101 & 1.06 & 13.0 \\
\hline 11 & 64.316 & 0.61 & 12.5 \\
\hline 12 & 64.487 & 0.63 & 11.0 \\
\hline 13 & 64.608 & 0.49 & 11.6 \\
\hline 14 & 64.708 & 0.42 & 12.0 \\
\hline 15 & 64.789 & 0.35 & 12.0 \\
\hline 16 & 64.854 & 0.32 & 15.0 \\
\hline 17 & $(64.913)$ & $(0.30)$ & (13.0) \\
\hline $18 \dagger$ & $(64.956)$ & $(0.74)$ & $(17.0)$ \\
\hline $19 \ddagger$ & $(64.995)$ & $(3.50)$ & $(35.0)$ \\
\hline 20 & $(65.029)$ & $(0.66)$ & $(13.0)$ \\
\hline$\vdots$ & - & - & - \\
\hline$\infty$ & 65.332 & - & - \\
\hline \multicolumn{4}{|c|}{$\begin{array}{c}N e^{2+}\left(2 s^{2} 2 p^{4}\right)^{1} D_{2} \rightarrow N e^{3+}\left(2 s^{2} 2 p^{3}\right)^{2} P_{1 / 2,3 / 2} \\
\delta=0.07\end{array}$} \\
\hline $6^{*}$ & $(64.487)$ & $(0.73)$ & $(12.0)$ \\
\hline 7 & 65.414 & 0.88 & 12.0 \\
\hline 8 & $(66.019)$ & $(0.47)$ & (13.0) \\
\hline 9 & 66.428 & 0.42 & 12.0 \\
\hline 10 & 66.721 & 0.33 & 11.0 \\
\hline 11 & 67.111 & 0.45 & 17.0 \\
\hline $12 \dagger \dagger$ & $(67.221)$ & $(0.35)$ & $(15.0)$ \\
\hline 13 & $(67.335)$ & $(0.09)$ & $(9.0)$ \\
\hline$\vdots$ & - & - & - \\
\hline$\infty$ & 67.962 & - & - \\
\hline
\end{tabular}

Table 2: Gaussian-fit centers $\left(E_{n}\right)$ for series labelled by I, II and III in Fig. 1, $n$ is the principal quantum number. Oscillator strengths $I$ and widths $\omega$ (approximately 0.849 meV FWHM) are also given. Values tabulated within brackets are uncertain. Some peaks superimpose between series: $\dagger$ with the $n=6$ peak of series VI; $\ddagger$ with the $n=6$ peak of series $\mathrm{V}$; * with the $n=12$ peaks of series II; $\dagger \dagger$ with the $n=10$ peaks of series $\mathrm{V}$. 


\begin{tabular}{|c|c|c|c|}
\hline$n$ & $\begin{array}{c}E_{n} \\
(\mathrm{eV})\end{array}$ & $\begin{array}{c}I \\
(e V \cdot M b)\end{array}$ & $\begin{array}{c}\omega \\
(m e V)\end{array}$ \\
\hline \multicolumn{4}{|c|}{$\begin{aligned} & \text { IV } \\
N e^{2+}\left(2 s^{2} 2 p^{4}\right)^{3} P_{2} & \rightarrow N e^{3+}\left(2 s^{2} 2 p^{3}\right)^{2} D_{5 / 2}^{\circ} \\
\delta & =0.05\end{aligned}$} \\
\hline 5 & $\begin{array}{l}63.522 \\
\end{array}$ & 1.38 & 10.0 \\
\hline 6 & 65.069 & 1.12 & 11.0 \\
\hline 7 & 65.989 & 0.54 & 11.0 \\
\hline 8 & 66.586 & 1.16 & 15.7 \\
\hline 9 & 67.006 & 0.30 & 11.3 \\
\hline 10 & 67.292 & 0.87 & 14.4 \\
\hline 11 & 67.506 & 1.05 & 12.0 \\
\hline 12 & $(67.673)$ & 0.31 & 13.6 \\
\hline$\vdots$ & - & - & - \\
\hline$\infty$ & 68.536 & - & - \\
\hline \multicolumn{4}{|c|}{$\begin{array}{c}N e^{2+}\left(2 s^{2} 2 p^{4}\right)^{3} P_{1} \rightarrow N e^{3+}\left(2 s^{2} 2 p^{3}\right)^{2} D_{5 / 2,3 / 2}^{\circ} \\
\delta=0.04\end{array}$} \\
\hline 5 & $(63.463)$ & $(0.20)$ & $(9.0)$ \\
\hline $6 \ddagger$ & (64.995) & $(3.50)$ & $(35.0)$ \\
\hline 7 & $(65.908)$ & $(0.11)$ & $(9.0)$ \\
\hline 8 & $(66.508)$ & $(0.18)$ & $(9.0)$ \\
\hline 9 & - & - & . \\
\hline $10 \dagger \dagger$ & $(67.221)$ & $(0.35)$ & $(15.0)$ \\
\hline 11 & 67.429 & 0.35 & 14.0 \\
\hline $12 \sharp$ & $(67.600)$ & $(0.42)$ & $(32.0)$ \\
\hline 13 & 67.731 & 0.07 & 9.4 \\
\hline$\vdots$ & - & - & - \\
\hline$\infty$ & 68.536 & - & - \\
\hline \multicolumn{4}{|c|}{$\begin{array}{c}N e^{2+}\left(2 s^{2} 2 p^{4}\right)^{3} P_{0} \rightarrow N e^{3+}\left(2 s^{2} 2 p^{3}\right)^{2} D_{5 / 2,3 / 2}^{\circ} \\
\delta=0.06\end{array}$} \\
\hline $6 \dagger$ & $(64.956)$ & $(0.74)$ & $(17.0)$ \\
\hline 7 & 65.890 & 0.17 & 13.0 \\
\hline 8 & 66.475 & 0.18 & 14.0 \\
\hline 9 & 67.188 & 0.06 & 7.0 \\
\hline 10 & 67.398 & 0.06 & 7.0 \\
\hline : & - & - & - \\
\hline$\infty$ & 68.422 & - & - \\
\hline
\end{tabular}

Table 3: Gaussian-fit centers $\left(E_{n}\right)$ for series labelled by IV, V and VI in Fig. 1 $n$ is the principal quantum number. Oscillator strengths $I$ and widths $\omega$ (approximately 0.849 meV FWHM) are also given. Values tabulated within brackets are uncertain. Some peaks superimpose between series: $\ddagger$ overlaps with the $n=19$ peak of series II. $\dagger \dagger$ overlaps with the $n=12$ peak of series III. $\sharp$ This center was derived from a double Gaussian function fit, the assignment corresponds to the smaller peak (see Fig. 1) while a large peak at $67.612 \mathrm{eV}$ remains unassigned. $\dagger$ Overlaps with series II, $n=18$ peak. 


\begin{tabular}{|c|c|c|c|}
\hline$n$ & $\begin{array}{c}E_{n} \\
(\mathrm{eV})\end{array}$ & $\begin{array}{c}I \\
(e V \cdot M b)\end{array}$ & $\begin{array}{c}\omega \\
(m e V)\end{array}$ \\
\hline \multicolumn{4}{|c|}{ VII } \\
\hline \multicolumn{4}{|c|}{$\begin{aligned} N e^{2+}\left(2 s^{2} 2 p^{4}\right)^{3} P_{2} & \rightarrow N e^{3+}\left(2 s^{2} 2 p^{3}\right)^{2} P_{1 / 2,3 / 2}^{\circ} \\
\delta & =0.075\end{aligned}$} \\
\hline 7 & 68.611 & 0.32 & 17 \\
\hline 8 & 69.215 & 0.22 & 14 \\
\hline 9 & 69.628 & 0.18 & 13 \\
\hline 10 & 69.924 & 0.14 & 12 \\
\hline 11 & 70.141 & 0.10 & 11 \\
\hline 12 & $(70.307)$ & $(0.08)$ & (13) \\
\hline 13 & $(70.433)$ & $(0.05)$ & (13) \\
\hline 14 & $(70.538)$ & $(0.08)$ & (13) \\
\hline$\vdots$ & - & - & - \\
\hline$\infty$ & 71.165 & - & - \\
\hline \multicolumn{4}{|c|}{$N e^{2+}\left(2 s^{2} 2 p^{4}\right)^{3} P_{1} \rightarrow N e^{3+}\left(2 s^{2} 2 p^{3}\right)^{2} P_{1 / 2,3 / 2}^{\circ}$} \\
\hline 7 & 68.531 & 0.19 & 16 \\
\hline 8 & 69.134 & 0.17 & 16 \\
\hline 9 & 69.548 & 0.12 & 13 \\
\hline 10 & 69.844 & 0.08 & 12 \\
\hline 11 & 70.061 & 0.01 & 11 \\
\hline 12 & 70.226 & 0.05 & 11 \\
\hline 13 & $(70.355)$ & $(0.05)$ & (12) \\
\hline$\vdots$ & - & - & - \\
\hline$\infty$ & 71.085 & - & - \\
\hline \multicolumn{4}{|c|}{$N e^{2+}\left(2 s^{2} 2 p^{4}\right)^{3} P_{0} \rightarrow N e^{3+}\left(2 s^{2} 2 p^{3}\right)^{2} P_{1 / 2,3 / 2}^{\circ}$} \\
\hline 7 & 68.497 & 0.04 & 12 \\
\hline 8 & 69.100 & 0.02 & 11 \\
\hline 9 & 69.515 & 0.05 & 14 \\
\hline 10 & $(69.810)$ & $(0.06)$ & $(22)$ \\
\hline$\vdots$ & - & - & - \\
\hline$\infty$ & 71.051 & - & - \\
\hline
\end{tabular}

Table 4: Gaussian-fit centers $\left(E_{n}\right)$ for series labelled by VII in Fig. 1 and VIII in Fig. 2 , $n$ is the principal quantum number. Oscillator strengths $I$ and widths $\omega$ (approximately $0.849 \mathrm{meV}$ FWHM) are also given. Values tabulated within brackets are uncertain. 


\begin{tabular}{|c|c|c|c|}
\hline$n \ell$ & $\begin{array}{c}E_{n} \\
(\mathrm{eV})\end{array}$ & $\begin{array}{c}I \\
(\mathrm{eV} \cdot \mathrm{Mb})\end{array}$ & $\begin{array}{c}\omega \\
(m e V)\end{array}$ \\
\hline \multicolumn{4}{|c|}{$\begin{array}{c}N e^{2+}\left(2 s 2 p^{5}\right)^{3} P_{2}^{\circ} \rightarrow N e^{3+}\left(2 s 2 p^{4}\right)^{4} P \\
\delta=0.47\end{array}$} \\
\hline 4 & 76.446 & 0.50 & 56 \\
\hline 5 & 80.321 & 0.71 & 92 \\
\hline 6 & 82.259 & 0.62 & 75 \\
\hline 7 & 83.384 & 0.24 & 65 \\
\hline 8 & 84.083 & 0.20 & 64 \\
\hline 9 & 84.552 & 0.14 & 62 \\
\hline$\vdots$ & - & - & - \\
\hline$\infty$ & 86.254 & - & - \\
\hline \multicolumn{4}{|c|}{$\delta=0.4$} \\
\hline $4(\delta=0.44)$ & 81.823 & 0.56 & 53 \\
\hline $4(\delta=0.48)$ & 82.020 & 0.33 & 46 \\
\hline $5(\delta=0.44)$ & $(85.735)$ & $(0.52)$ & $(70)$ \\
\hline $5(\delta=0.48)$ & $(85.816)$ & $(0.15)$ & $(42)$ \\
\hline 6 & $(87.718)$ & $(0.44)$ & $(82)$ \\
\hline 7 & $(88.878)$ & $(0.12)$ & $(45)$ \\
\hline 8 & $(89.608)$ & $(0.13)$ & (39) \\
\hline 9 & $(90.089)$ & $(0.06)$ & (18) \\
\hline 10 & $(90.430)$ & $(0.05)$ & $(21)$ \\
\hline 11 & $(90.675)$ & $(0.03)$ & $(22)$ \\
\hline 12 & $(90.858)$ & $(0.06)$ & $(23)$ \\
\hline 13 & $(91.004)$ & $(0.03)$ & (18) \\
\hline$\vdots$ & - & - & - \\
\hline$\infty$ & 91.723 & - & - \\
\hline
\end{tabular}

Table 5: Gaussian-fit centers $\left(E_{n}\right)$ for series labelled by IX in Fig. 2, $n$ is the principal quantum number. Oscillator strengths $I$ and widths $\omega$ (approximately $0.849 \mathrm{meV}$ FWHM) are also given. Values tabulated within brackets are uncertain. ${ }^{\gamma}$ This value of $\delta$ corresponds to an average of both series. 


\section{References}

[1] A. Bartnik, P. Wachulak, T. Fok, L. Wegrzynski, H. Fiedorowicz, T. Pisarczyk, T. Chodukowski, Z. Kalinowska, R. Dudzak, J. Dostal, E. Krousky, J. Skala, J. Ullschmied, J. Hrebicek, and T. Medrik. Photoionized plasmas induced in neon with extreme ultraviolet and soft $\mathrm{x}$-ray pulses produced using low and high energy laser systems. Physics of Plasmas, 22:043302, 2015.

[2] Yuri Ralchenko. Modern Methods in Collisional-Radiative Modeling of Plasmas. Yuri Ralchenko, National Institute of Standards and Technology Gaithersburg, MD USA, 2016.

[3] J. E. Bailey, T. Nagayama, G. P. Loisel, G. A. Rochau, C. Blancard, J. Colgan, Ph. Cosse, G Faussurier, C. J. Fontes, F. Gilleron, I. Golovkin, C. Hansen, S. B. Iglesias, D. Kilcrease, J MacFarlane, R. C Mancini, S. N. Nahar, J.-C. Orban, C. Pain, A. K Pradhan, M. Sherrill, and B. G. Wilson. A higher-than-predicted measurement of iron opacity at solar interior temperatures. Nature, pages 56-59, 2015.

[4] Bon-Chul Koo, Yong-Hyun Lee, Dae-Sik Moon, Sung-Chul Yoon, and John C. Raymond. Phosphorus in the young supernova remnant cassiopeia A. Science, 342:1346-1348, 2013.

[5] A. E. Jaskot and S. Ravindranath. Photoionization models for the semiforbidden C III] 1909 emission in star-forming galaxies. Astrophys. J., 833:136, 2016.

[6] Roland Thissen, Olivier Witasse, Odile Dutuit, Cyril Simon Wedlund, Guillaume Gronoff, and Jean Lilensten. Doubly-charged ions in the planetary ionospheres: a review. Phys. Chem. Chem. Phys., 13:1826418287, 2011.

[7] Henrik Kjeldsen. Photoionization cross sections of atomic ions from merged-beam experiments. J. Phys. B. At. Mol. Opt., 39:R325, 2006.

[8] Stefan Schippers, A. L. David Kilcoyne, Ronald A. Phaneuf, and Alfred Müller. Photoionisation of ions with synchrotron radiation: from ions in space to atoms in cages. Contemp. Phys., 57:215-229, 2016. 
[9] J B West. Photoionization of atomic ions. J. Phys. B. At. Mol. Opt., 34:R45, 2001.

[10] M. A. Shure, J. R. Houck, G. E. Gull, and T. Herter. Detection of the Ne III 36 micron forbidden line in the planetary nebula ngc 6543. Astrophys. J., 281:L29-L31, 1984.

[11] A K Pradhan. Close-coupling calculations for photoionisation cross sections of neon ions: Ne II, Ne III and Ne IV. J Phys. B. At. Mol. Opt., 12:3317, 1979 .

[12] A. K. Pradhan. Photo-ionization cross-sections of Ne II, Ne III and Ne IV. Mon. Not. R. Astron Soc., 190:5P-8P, 1980.

[13] The Opacity Project Team. The Opacity Project, volume 1 and 2. Institute of Physics Publishing, 1996.

[14] Claudio Mendoza, Claude Zeippen, Anil Pradhan, and Franck Delahaye. Topbase, 2018.

[15] A. M. Covington, A. Aguilar, I. R. Covington, M. F. Gharaibeh, G. Hinojosa, C. A. Shirley, R. A. Phaneuf, I. Alvarez, C. Cisneros, I. Dominguez-Lopez, M. M. Sant'Anna, A. S. Schlachter, B. M. McLaughlin, and A. Dalgarno. Photoionization of $\mathrm{Ne}^{+}$using synchrotron radiation. Phys. Rev. A, 66:062710, 2002.

[16] A Aguilar, E D Emmons, M F Gharaibeh, A M Covington, J D Bozek, G Ackerman, S Canton, B Rude, A S Schlachter, G Hinojosa, I Alvarez, C Cisneros, B M McLaughlin, and R A Phaneuf. Photoionization of ions of the nitrogen isoelectronic sequence: experiment and theory for $F^{2+}$ and $\mathrm{Ne}^{3+}$. J. Phys. B. At. Mol. Opt., 38:343, 2005.

[17] M. Oura, H. Yamaoka, Y. Senba, H. Ohashi, and F. Koike. Observation of the $[1 s 2 s]^{3,1} \operatorname{Snln}^{\prime} l^{\prime}{ }^{1} P$ inner-shell doubly excited states of ne by photoion yield spectroscopy. Phys. Rev. A, 70:062502, 2004.

[18] H. Yamaoka, M. Oura, K. Kawatsura, T. Hayaishi, T. Sekioka, A. Agui, A. Yoshigoe, and F. Koike. Photoionization of singly and doubly charged neon ions following inner-shell excitation. Phys. Rev. A, 65:012709, 2001. 
[19] Alfred Müller, Dietrich Bernhardt, Alexander Borovik Jr., Ticia Buhr, Jonas Hellhund, Kristof Holste, A. L. David Kilcoyne, Stephan Klumpp, Michael Martins, and Sandor Ricz. Photoionization of Ne atoms and $\mathrm{Ne}^{+}$ions near the K edge: Precision spectroscopy and absolute crosssections. Astrophys. J., 836:166, 2017.

[20] Jiaolong Zeng and Jianmin Yuan. Photoionization of Be-like neon (Ne VII) from the low-lying states: Energies, widths, branching ratios, and oscillator strengths of the $1 s-2 p$ resonances. Phys. Rev. A, 66:022715, 2002.

[21] Dae-Soung Kim and Duck-Hee Kwon. Photoionization of Be-like $\mathrm{N}^{3+}$ and $\mathrm{Ne}^{6+}$ ions: features of $\mathrm{Be}$ isoelectronic sequences of $\mathrm{Z} \leq 10$. J. Phys. B-At. Mol. Opt., 45:185201, 2012.

[22] G. C. Angel and James A. R. Samson. Total photoionization cross sections of atomic oxygen from threshold to 44.3 A. Phys. Rev. A, 38:5578-5585, 1988.

[23] Sultana N. Nahar. Photoionization cross sections and oscillator strengths for oxygen ions: OI-OVII. Phys. Rev. A, 58:3766-3782, 1998.

[24] R. Flesch, M. C. Schürmann, J. Plenge, H. Meiss, M. Hunnekuhl, and E. Rühl. Autoionization and photoionization of $O\left({ }^{1} D\right)$. Phys. Rev. A, 62:052723, 2000 .

[25] S. S. Tayal. Resonant photoionization cross sections and branching ratios for atomic oxygen. Phys. Rev. A, 65:032724, 2002.

[26] Leyla Eser, Seldaand Özdemir. E1, E2 and M1 transition parameters for some levels over ionization limit of Ne III. Eur. Phys. J. Plus, 131:227, 2016.

[27] Anil K. Pradhan and Sultana S. N. Nahar. Atomic astrophysics and spectroscopy. Cambridge University Press, 2011.

[28] PG Burke and WD Robb. The R-matrix theory of atomic processes. Advances in atomic and molecular physics, 11:143-214, 1976.

[29] M. J. Seaton. Atomic data for opacity calculations. I. General description. J. Phys. B, 20:6363, 1987. 
[30] K. A. Berrington, P. G. Burke, K. Butler, M. J. Seaton, P. J. Storey, K. T. Taylor, and Y. Yan. Atomic data for opacity calculations. II. Computational methods. J. Phys. B, 20:6379, 1987.

[31] Keith A. Berrington, Werner B. Eissner, and Patrick H. Norrington. RMATRX1: Belfast atomic R-matrix codes. Comput. Phys. Commun., 92:290 - 420, 1995.

[32] Sultana N. Nahar and Anil K. Pradhan. Unified treatment of electronion recombination in the close-coupling approximation. Phys. Rev. A, 49:1816-1835, 1994.

[33] H L Zhang, Sultana N Nahar, and Anil K Pradhan. Close-coupling $\mathrm{R}$-matrix calculations for electron-ion recombination cross sections. $J$. Phys. B, 32:1459, 1999.

[34] W. Eissner, M. Jones, and H. Nussbaumer. Techniques for the calculation of atomic structures and radiative data including relativistic corrections. Comput. Phys. Commun., pages 270 - 306, 1974.

[35] Sultana N. Nahar, Werner Eissner, Guo-Xin Chen, and Anil K. Pradhan. Atomic data from the iron project: LIII. Relativistic allowed and forbidden transition probabilities for Fe XVII. Astron. Astrophys., 408:789801, 2003.

[36] A. Kramida, Yu. Ralchenko, J. Reader, and and NIST ASD Team. NIST Atomic Spectra Database (ver. 5.5.6), [Online]. Available: http://physics.nist.gov/asd [Fri Aug 03 2018]. National Institute of Standards and Technology, Gaithersburg, MD., 2018.

[37] A Müller, S Schippers, J Hellhund, K Holste, A L D Kilcoyne, R A Phaneuf, C P Ballance, and B M McLaughlin. Single-photon single ionization of $\mathrm{W}^{+}$ions: experiment and theory. J. Phys. B, 48:235203, 2015 .

[38] G. Hinojosa, V. T. Davis, A. M. Covington, J. S. Thompson, A. L. D. Kilcoyne, A. Antillón, E. M. Hernández, D. Calabrese, A. Morales-Mori, A. M. Juárez, O. Windelius, and B. M. McLaughlin. Single photoionization of the $\mathrm{Zn}$ II ion in the photon energy range 17.5 to $90.0 \mathrm{eV}$ : experiment and theory. Mon. Not. R. Astron Soc., 470:4048-4060, 2017. 
[39] George C King, Michel Tronc, Frank H Read, and Richard C Bradford. An investigation of the structure near the $\mathrm{L}_{2,3}$ edges of argon, the $\mathrm{M}_{4,5}$ edges of kryton and the $\mathrm{N}_{4,5}$ edges of xenon, using electron impact with high resolution. Jour. Phys. B., 10:2479-2495, 1977.

[40] M. Domke, K. Schulz, G. Remmers, G. Kaindl, and D. Wintgen. Highresolution study of ${ }^{1} \mathrm{P}^{o}$ double-excitation states in helium. Phys. Rev. A, 53:1424-1438, 1996.

[41] R. E. Huffman, J. C. Larrabee, and Y. Tanaka. New absorption spectra of atomic and molecular oxygen in the vacuum ultraviolet. II. rydberg series from $O I\left({ }^{1} D_{2}\right)$ and $O I\left({ }^{1} S_{0}\right)$ metastable states. J. Chem. Phys., 47:4462-4471, 1967.

[42] R. E. Huffman, J. C. Larrabee, and Y. Tanaka. New absorption spectra of atomic and molecular oxygen in the vacuum ultraviolet. I. Rydberg series from O I ground state and new excited $O_{2}$ bands. J. Chem. Phys., 46:2213-2233, 1967. 